\title{
Alleviation of medial meniscal transection-induced osteoarthritis pain in rats by human adipose derived mesenchymal stem cells
}

\author{
Zhifeng Wang ${ }^{1,2}$, Hao Zhu ${ }^{1}$, Shuhang Dai ${ }^{1}$, Ke Liu ${ }^{1}$, Chenxi Ge ${ }^{1}$ \\ ${ }^{1}$ Sinoneural Cell Engineering Group Co., Ltd., Shanghai, China; ${ }^{2}$ Translational Medical Center for Stem Cell Therapy and Institute for Regenerative \\ Medicine, Shanghai East Hospital, Tongji University, Shanghai, China \\ Correspondence to: Dr. Zhifeng Wang. Sinoneural Cell Engineering Group Co., Ltd., Shanghai, China. Email: wangzhifeng68@hotmail.com.
}

\begin{abstract}
Knee osteoarthritis (KOA) is a degenerative joint disorder manifested with deformity, pain, and functional disability due to damage of the articular cartilage. Cell therapy with mesenchymal stem cells (MSCs) holds great promise to alleviate or even cure the degenerative diseases including KOA. However, the evidence of efficacy of human adipose tissue-derived MSCs (hAdMSCs) on KOA therapy remains limited. Here, we evaluate the therapeutic efficacy of hAdMSCs for KOA, using a medial meniscal transection (MMT) rat model. Our study demonstrated that intra-articular injection of $1.25 \times 10^{6}$ hAdMSCs significantly attenuated MMT-induced joint pain in a KOA rats model. The results of this study provide strong evidence that hAdMSCs-based therapy can be regarded as a prominent treatment option for patients with KOA.
\end{abstract}

Keywords: Osteoarthritis; adipose mesenchymal stem cell; xenotransplantation; joint pain

Received: 19 January 2020; Accepted: 09 June 2020; Published: 17 June 2020.

doi: 10.21037/sci-2020-003

View this article at: http://dx.doi.org/10.21037/sci-2020-003

\section{Introduction}

Osteoarthritis (OA) is the most common form of arthritis, and current prevalence is $12 \%$ in the population over 60 years old, which will escalate over the next 20 years (1). Characteristic features of $\mathrm{OA}$ include chronic degeneration of articular cartilage, matrix fibrillation, erosion, sclerosis of subchondral bone and osteophyte formation (2). Although it is widely accepted that $\mathrm{OA}$ is a degenerative condition of the cartilage, both subchondral bone and synovial membrane also actively participate in the progression of the disease (3). One of typical symptoms of KOA is pain, which can progress to be continuous, and exert a significant detrimental effect on the movement and quality of life (1). Currently available options for the treatment of KOA include physical and occupational therapy, weight loss, administration of nonsteroidal anti-inflammatory drugs (NSAID), modification of activity levels for daily living, intra-articular injections of hyaluronic acid, corticosteroids and platelet rich plasma (PRP), etc., joint surgery (4). However, these therapeutic strategies for KOA only provide symptomatic relief, and cannot alter the natural progression of this disease in the clinic. Hence, there is an urgent need to develop new strategies to attenuate KOA development and relieve the pain induced by KOA.

Recent progress in the development of MSCs open the possibility of reversing OA-induced alternations, relying on MSCs' regenerative property along with their immunomodulatory and anti-inflammatory capacities. MSCs express the cell surface markers CD73, and CD90, CD105, but lack of expression of CD14, CD31, CD34, CD45, and HLA-DR. Furthermore, MSCs not only can spontaneously differentiate into osteocytes and osteoblasts in vivo, also could be induced to differentiate into adipocytes, chondrocytes, and osteoblasts in vitro (5). By examining these cell surface markers as well as multilineage differentiations potential, MSCs have been identified and isolated from various tissues, such as bone marrow, adipose tissue, umbilical cord, cord blood, amnion, placenta, amniotic fluid, and dental pulp (6). Compared with MSCs from bone marrow, hAdMSCs are becoming more popular for therapy due to ease of acquisition, high levels of multipotency, low immunogenicity, and high proliferative capacity (7). For these reasons, the 
therapeutic effects of hAdMSCs have been extensively investigated in animal models of different diseases such as nerve injuries, metabolic disorders, diabetes mellitus, and neurodegenerative disorders (8-11). For example, intravenous administration of cultured hAdMSCs improved glucose tolerance, increased $\beta$ cell proliferation, and preserved $\beta$ cell mass in STZ-treated NOD-SCID mice (10). As another example, co-transplanting mouse neural stem cells (mNSCs) and hAdMSCs significantly increased the viability of mNSCs in a rat spinal cord injury model, indicating that this novel strategy may be a more effective therapeutic strategy to treat this disease (12). Nevertheless, the evidence supporting successful reversion of $\mathrm{KOA}$ in vivo via hAdMSCs administration remains limited.

In the present in vivo study, we examined the beneficial effects of cell-based therapy for the alleviation of joint pain by intra-articular injection of $1.25 \times 10^{6} \mathrm{hAdMSCs}$ in MMTinduced KOA rats model. The transplantation of human cells in rats made it possible to avoid the largely unexplained problems encountered in culture-expanded murine MSCs $(13,14)$, and to examine the cells that are the most relevant for potential clinical trials in patients with KOA. Here we reported an impressively positive therapeutic effects of hAdMSCs-based therapy for KOA, which shed light on their potential clinical application in the future.

\section{Methods}

\section{bAdMSCs isolation, culture and characterization}

Human adipose tissue was obtained through elective liposuction with informed consent. Isolation and expansion of adipose derived MSCs will be undertaken according to previously published techniques (15). Briefly, lipoaspirate was transferred into 50-ml tube for centrifugation at $400 \mathrm{~g}$ for $5 \mathrm{~min}$. After digestion with collagenase I solution and filtration through a $100-\mu \mathrm{m}$ filter, stromal vascular fraction (SVF) was obtained. Cells were cultured in $175 \mathrm{~cm}^{2}$ flask until the third passage for cell therapy.

Culture-expanded of cells at passage five were analyzed with flow cytometry to detect the expressions of cell surface markers. The multi-lineage differentiations potential of hAdMSCs was evaluated using cell differentiation kits according to the manufacturer's instructions (MoBiTec., Lorzestrasse, Germany). The presence of adipocytes was identified by Oil Red O staining, chondrocytes by Alcian Blue staining, and osteocytes by Alizarin Red S staining.

\section{Animal studies}

Adult male Lewis rats weighing approximately $320 \mathrm{~g}$ were purchased from Charles River Beijing. The study was approved by the Laboratory Animal Care and Use Committee of Tongji University and animal care and experiments were performed in accordance with the National Institutes of Health Guide for the Care and Use of Laboratory Animals.

The right knee of rats was prepared for OA knee model induced by MMT surgery. After one week of recovery, the rats were randomly divided into two groups receiving intraarticular injection of vehicle or $1.25 \times 10^{6}$ hAdMSCs in $50 \mu \mathrm{L}$ saline solution. Spontaneous distribution of weight between the hind limbs was measured with an incapacitance meter (IITC Life Science, Woodland Hills, CA, USA) before and at defined timepoints during the 28-day period posthAdMSCs injection. Weight-bearing distribution (\%) $=$ [weight on the affected leg/(weight on the unaffected leg + weight on the affected leg)] $\times 100$.

\section{Data analysis and presentation}

Differences between two independent groups were analyzed with Student's $t$-test. Data are represented by mean \pm standard error. All statistical analyses and chart preparations were performed with GraphPad Prism 8 software (GraphPad Software, Inc., La Jolla, CA, USA). P values less than 0.05 were considered significant.

\section{Results}

In the present study, these cells showed a characteristic spindle-shaped and fibroblast-like morphology, and were attached to the plate during cell culture (Figure 1A). To examine multiple differentiation potential of hAdMSCs, culture-expanded cells at passage three were induced to differentiate into adipocytes as assessed by Oil Red $\mathrm{O}$ staining (Figure $1 B$ ), osteoblasts as assessed by Alizarin Red S staining (Figure 1C), chondroblasts as assessed by Alcian Blue staining (Figure 1D). The results of FACS analysis showed that $100 \%$ of the hAdMSCs positively express CD44, CD73, CD90, CD105, and CD166, whereas less than $0.2 \%$ were positive for CD14, CD19, CD34, CD45, and HLA-DR (Figure 1E). To minimize the risk of contamination, the isolation and culture of hAdMSCs were performed in a GMP-grade clean room facility, and 

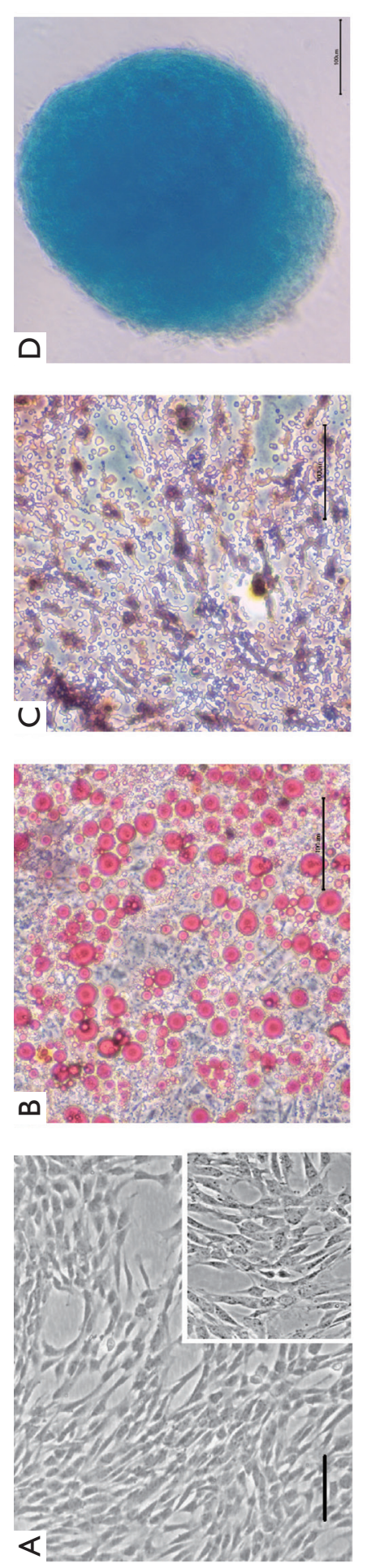
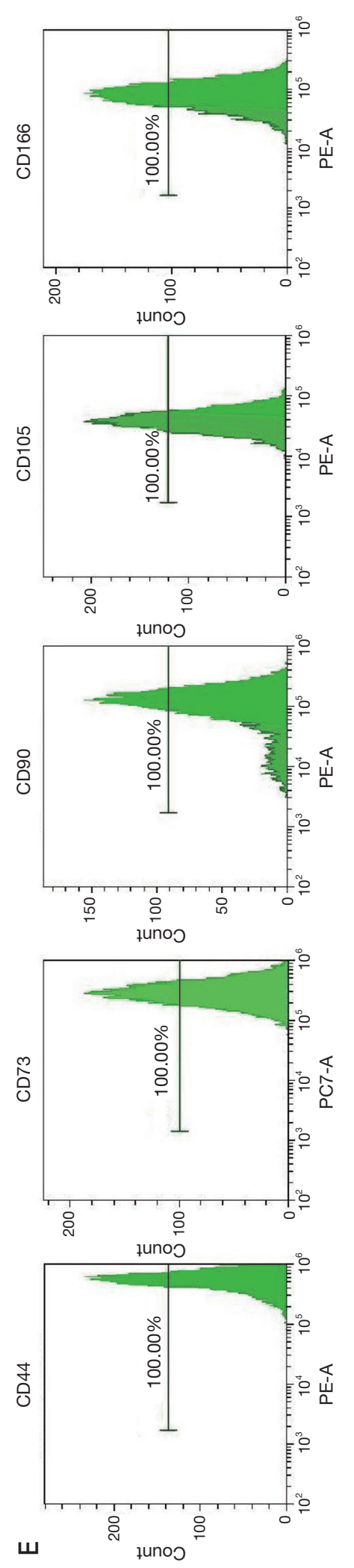

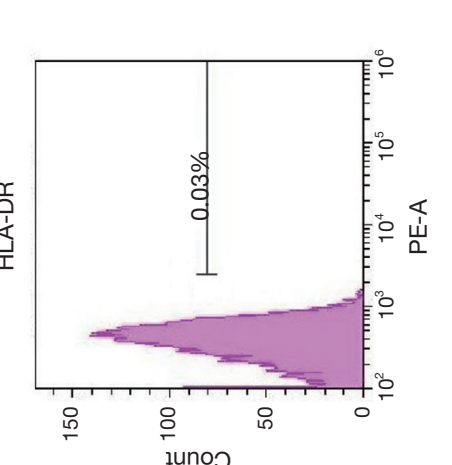

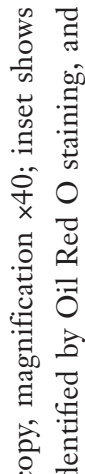
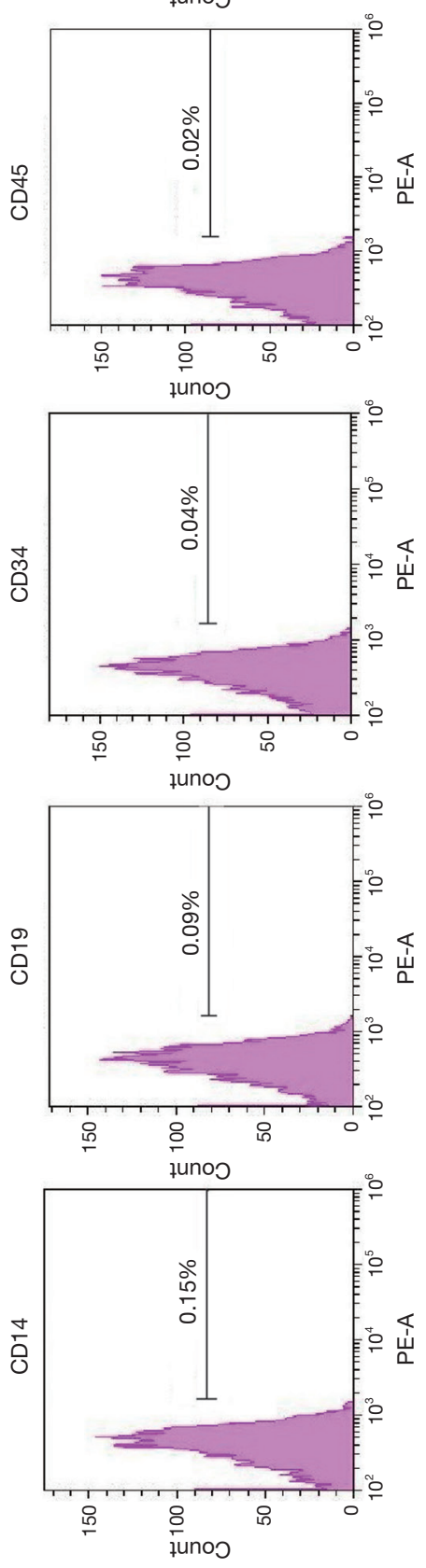

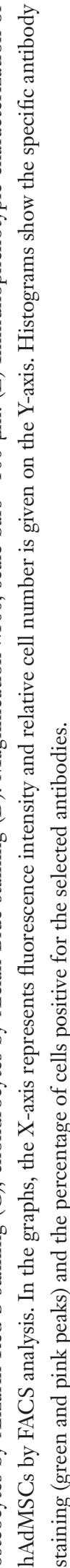


A
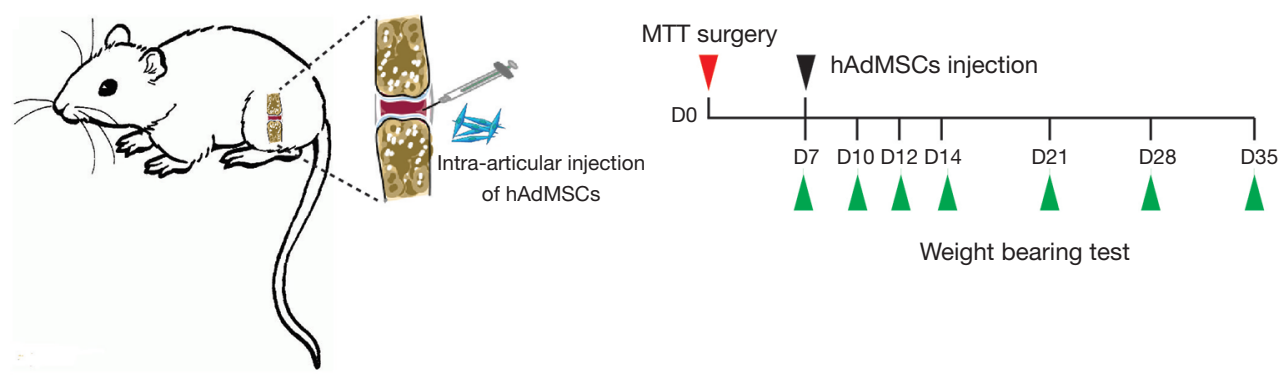

Weight bearing test

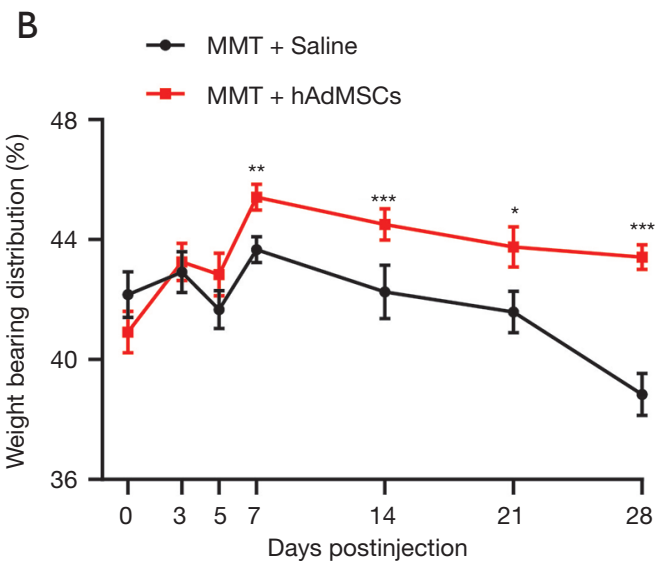

C

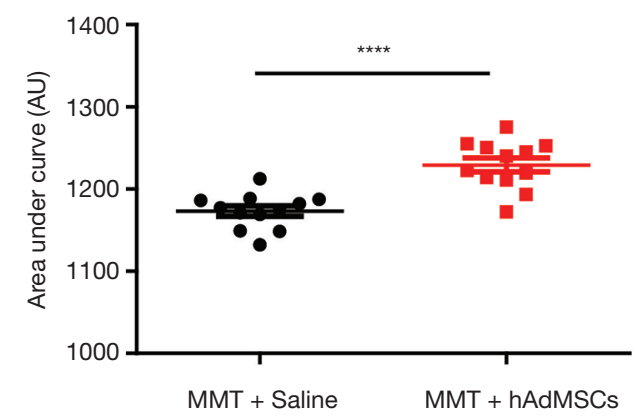

Figure 2 hAdMSCs therapy attenuated joint pain in MTT-induced KOA rats. (A) Schematic of the time course used for the in vivo KOA experiments. D, day. (B) Rats receiving hAdMSCs exhibited an attenuated response to MTT-induced KOA pain. Statistical comparison between two groups at each timepoint was performed with Student's $t$-test. (C) Area under the curve (AUC) data calculated for days 3, 5, 7, 14, 21, and 28 postinjection revealed a significant increasement in spontaneous weight-bearing on the affected right hind limbs of MTT rats after receiving hAdMSCs injection. Comparison of AUC used a two-tailed unpaired Student's $t$-test. ${ }^{*} \mathrm{P}<0.05,{ }^{* *} \mathrm{P}<0.01,{ }^{* * *} \mathrm{P}<0.001$, ${ }^{* * * *} \mathrm{P}<0.0001$. Data are mean $\pm \mathrm{SEM}, \mathrm{n}=12$ rats in each group.

the preclinical and/or clinical lot was produced under strict GMP conditions. These cells were negative for fungi, bacteria, and mycoplasmas, and cell viability determined by trypan blue exclusion was $>95 \%$ before cell infusion (data available upon request from the corresponding author).

In the present study, rats KOA was surgically induced by medial meniscal transection, a validated preclinical model of KOA pain that includes cartilage degradation, proteoglycan loss, and osteophyte formation, which was similar to those that occur in human OA (2). Because of the acute injury, cartilage degradation and development of joint pain are typical features encountered in MMT-induced KOA. The latter is usually determined by an incapacitance meter in the so-called weight-bearing test (16). Because of MSCs' regenerative capacity along with their trophic effects, we investigated whether hAdMSCs could alleviate the development of the MMT-induced joint pain. hAdMSCs $\left(1.25 \times 10^{6}\right.$ cells $)$ were injected into the articular cavity of right joints on day 7 post MMT surgery, and weightbearing tests were performed at indicated timepoints (Figure 2A). After MMT surgery, there was a fall in the spontaneous weight-bearing on the surgical joint in vehicle-injection rats (Figure 2B,C), indicative of joint pain. However, an attenuated response was observed in hAdMSCs-injection rats as seen in Figure 2B,C, suggesting that hAdMSCs administration efficiently prevent the development of osteoarthritis-like joint pain induced by MMT. No significant difference was found in body weight between the two groups over four weeks (data available upon request from the corresponding author).

\section{Discussion}

The current study successfully isolated hAdMSCs from lipoaspirate tissue samples, which is confirmed by flow cytometry and assessment of tri-lineage differentiation 
potential. The hAdMSCs showed specific cell surface marker CD44, CD73, CD90, and CD105, and the absence of hematopoietic (CD45) and endothelial (CD34) antigens, which is consistent with the expression profiles of MSCs from bone marrow and umbilical cord (17-20). These results also showed that the isolated MSCs were not derived from hematopoietic or endothelial cells.

MSCs-based therapies for cartilage regeneration are extensively investigated, in both the pre-clinical and clinical environment. However, the therapeutic effect of xenogeneic AdMSCs transplantation on OA remains to be determined. It is natural that AdMSCs allotransplantation is more practical and rational than xenotransplantation in the treatment of KOA. However, it should be considered that xenotransplantation with hAdMSCs is a prerequisite for the ultimate therapeutic goal, which is potential clinical trials conducted in an ethical and safe manner. AdMSCs are considered as nonimmunogenic because they do not express histocompatibility complexII (MHC-II). Additionally, AdMSCs possess strong immunosuppressive properties mediated by cell-cell contact or by their paracrine factors such as transforming growth factor- $\beta 1$ (TGF- $\beta 1$ ) and prostaglandin E2 (PGE2) $(21,22)$. All of these characteristics allows these cells to escape recognition by $\mathrm{CD} 4+\mathrm{T}$ cells and protects them from natural killer (NK) cell-mediated deletion (23), which permits allogeneic or even xenogeneic AdMSCs transplanted into immunocompetent recipients without immunosuppressants. A number of studies have demonstrated excellent therapeutic effects of hAdMSCs in animal models of different diseases such as tendon injury, multiple sclerosis, acute anal sphincter injury, and bone defects without adverse side effects, thus indicating immunocompatibility of these cells (24-27). Therefore, we evaluated the effectiveness of hAdMSCs xenotransplantation in articular cartilage preservation in a rat KOA model, as they appear to be a good source for cartilage regeneration (28). Our results showed that single intra-articular injection of $1.25 \times 10^{6} \mathrm{hAdMSCs}$ significantly attenuated MMT-induced joint pain, which shed light their clinical applications for the treatment of patients with KOA. The xenotransplantation of hAdMSCs into immune-competent rats carries a risk of triggering an immune response. Here, we found that all of the rats were viable and had no side effects during the 28-day period post-hAdMSCs injection, suggesting these human cells may be immunoprivileged even xenotransplanted into immunocompetent recipients. For the KOA patients who are not suitable to harvest autologous MSCs, allogenic AdMSCs may therefore be an alternative choice.

Long-term persistence and/or proliferation of the human MSCs (hMSCs) in the rat joint is essential in order to exert their effects on promoting cartilage protection and pain alleviation. Although a number of preclinical investigations have demonstrated the efficacy of intraarticular injection of MSCs for the treatment of OA, the fate of the xenotransplanted human cells is still under debate. CM-DiI labeled $2 \times 10^{6}$ human bone marrow MSCs (hBMSCs) were injected into the rat's OA joint, and labeled cells cannot be detected at day 7 with the method of RT-PCR for human GAPDH, but still detected at 8 weeks by immunohistochemistry (29). McKinney et al. found complete loss of hBMSCs bioluminescent signal in the rat knee joint at day 7 post-injection (30). In another report, $\mathrm{Li}$ and colleagues found that fluorescent signals of injected DiD-labeled $2.5 \times 10^{6}$ hAdMSCs were detectable up to 70 days post-injection (31), which was consistent with the time for which we observed efficacy at 28 days post xenotransplantation of hAdMSCs. The survival discrepancies of injected hMSCs among existing reports probably arise from different experimental designs, such as labeling methods, cell source and number, duration of follow-up, or sensitivity of the technique adopted for cell tracking analysis. Supporting the concept that cell-therapy function as a living drug, Li et al. found that injected hAdMSCs were proliferative in rat meniscus and cartilage evidenced by positive antihuman ki67 signals, which can last for about 10 weeks (31). In addition to self-proliferation of hMSCs in rat knee joint, the results from Horie $e t$ al. showed that intra-articular injected hMSCs were activated to express Indian hedgehog (Ihh), bone morphogenetic protein 2 (BMP2), and parathyroid hormone-like hormone (PTHLH), which contributed to meniscal regeneration by stimulating chondrocyte proliferation (29).

Although present study demonstrated the efficacy of xenogeneic hAdMSCs transplantation for pain reduction, questions remain for a sound understanding of their mechanism of action. In addition to paracrine signaling, MSCs may act through direct engraftment in concert with the local environment $(29,32,33)$. However, it has been shown that only a small number of injected hMSCs engrafted to the regenerating edge up-regulates the expression of chondrogenic genes in rat cells, which indicated that the newly regenerated tissue derives mostly from the differentiation of the host cells $(29,33)$. These data suggest that the recruitment of endogenous 
cells may play a critical role in MSCs-based therapies, through paracrine communication (30). MSCs can secrete a variety of paracrine factors that aid in recruit of stem and progenitor cells, repair of degraded tissue and, most importantly, counteracting inflammation $(30,34)$. For example, in response to cartilage degeneration, MSCs can induce tissue remodeling by secreting multiple factors such as stromal-cell-derived factor (SDF)-1 and interleukin (IL)$6(35,36)$. MSCs can also mediate the immune-suppression by constitutively secreting prostaglandin-E2 (PGE2), which inhibits the proliferation of T- and natural killer (NK) cells but causes an increase in the pool of regulatory $\mathrm{T}$ (Treg) cells (34). McKinney et al. showed that encapsulation of hBMSCs with sodium alginate significantly attenuated MMT-induced cartilage degeneration, which indicated that MSCs could exert a chondroprotective therapeutic effect on early stage OA via paracrine signaling rather than direct engraftment (30).

Here, our results clearly show that intra-articular injection of hAdMSCs greatly alleviate OA-induced join pain. Nonetheless, the role of direct cellular engraftment versus paracrine action of hAdMSCs remains to be explored further to accelerate clinical translation of hAdMSCs-based therapies.

\section{Acknowledgments}

Thanks to all those who participated in this study. Funding: None.

\section{Footnote}

Conflicts of Interest: All authors have completed the ICMJE uniform disclosure form (available at http://dx.doi. org/10.21037/sci-2020-003). The authors have no conflicts of interest to declare.

Ethical Statement: The authors are accountable for all aspects of the work in ensuring that questions related to the accuracy or integrity of any part of the work are appropriately investigated and resolved. The study was approved by the Laboratory Animal Care and Use Committee of Tongji University and animal care and experiments were performed in accordance with the National Institutes of Health Guide for the Care and Use of Laboratory Animals.

Open Access Statement: This is an Open Access article distributed in accordance with the Creative Commons Attribution-NonCommercial-NoDerivs 4.0 International License (CC BY-NC-ND 4.0), which permits the noncommercial replication and distribution of the article with the strict proviso that no changes or edits are made and the original work is properly cited (including links to both the formal publication through the relevant DOI and the license). See: https://creativecommons.org/licenses/by-nc-nd/4.0/.

\section{References}

1. Glyn-Jones S, Palmer AJ, Agricola R, et al. Osteoarthritis. Lancet 2015;386:376-87.

2. Janusz MJ, Bendele AM, Brown KK, et al. Induction of osteoarthritis in the rat by surgical tear of the meniscus: inhibition of joint damage by a matrix metalloproteinase inhibitor. Osteoarthritis Cartilage 2002;10:785-91.

3. Kapoor M, Martel-Pelletier J, Lajeunesse D, et al. Role of proinflammatory cytokines in the pathophysiology of osteoarthritis. Nat Rev Rheumatol 2011;7:33-42.

4. Cheng JH, Wang CJ, Chou WY, et al. Comparison efficacy of ESWT and Wharton's jelly mesenchymal stem cell in early osteoarthritis of rat knee. Am J Transl Res 2019;11:586-98.

5. Shi Y, Wang Y, Li Q, et al. Immunoregulatory mechanisms of mesenchymal stem and stromal cells in inflammatory diseases. Nat Rev Nephrol 2018;14:493-507.

6. Hass R, Kasper C, Bohm S, et al. Different populations and sources of human mesenchymal stem cells (MSC): a comparison of adult and neonatal tissue-derived MSC. Cell Commun Signal 2011;9:12.

7. Wang M, Song L, Strange C, et al. Therapeutic effects of adipose stem cells from diabetic mice for the treatment of type 2 diabetes. Mol Ther 2018;26:1921-30.

8. Saidi R, Rajeshkumar R, Shariftabrizi A, et al. Human adipose-derived mesenchymal stem cells promote liver regeneration. J Invest Surg 2015;28:303-8.

9. Dasari VR, Veeravalli KK, Dinh DH. Mesenchymal stem cells in the treatment of spinal cord injuries: a review. World J Stem Cells 2014;6:120-33.

10. Kono TM, Sims EK, Moss DR, et al. Human adiposederived stromal/stem cells protect against STZ-induced hyperglycemia: analysis of hASC-derived paracrine effectors. Stem Cells 2014;32:1831-42.

11. Tajiri N, Acosta SA, Shahaduzzaman M, et al. Intravenous transplants of human adipose-derived stem cell protect the brain from traumatic brain injury-induced neurodegeneration and motor and cognitive impairments: 
cell graft biodistribution and soluble factors in young and aged rats. J Neurosci 2014;34:313-26.

12. Oh JS, Kim KN, An SS, et al. Cotransplantation of mouse neural stem cells (mNSCs) with adipose tissuederived mesenchymal stem cells improves mNSC survival in a rat spinal cord injury model. Cell Transplant 2011;20:837-49.

13. Baddoo M, Hill K, Wilkinson R, et al. Characterization of mesenchymal stem cells isolated from murine bone marrow by negative selection. J Cell Biochem 2003;89:1235-49.

14. Peister A, Mellad JA, Larson BL, et al. Adult stem cells from bone marrow (MSCs) isolated from different strains of inbred mice vary in surface epitopes, rates of proliferation, and differentiation potential. Blood 2004;103:1662-8.

15. Zuk PA, Zhu M, Mizuno H, et al. Multilineage cells from human adipose tissue: implications for cell-based therapies. Tissue engineering 2001;7:211-28.

16. Okun A, Liu P, Davis P, et al. Afferent drive elicits ongoing pain in a model of advanced osteoarthritis. Pain 2012;153:924-33.

17. Nakano A, Harada T, Morikawa S, et al. Expression of leukocyte common antigen (CD45) on various human leukemia/lymphoma cell lines. Acta Pathol Jpn 1990;40:107-15.

18. Miettinen M, Lindenmayer AE, Chaubal A. Endothelial cell markers CD31, CD34, and BNH9 antibody to $\mathrm{H}$ - and $\mathrm{Y}$-antigens--evaluation of their specificity and sensitivity in the diagnosis of vascular tumors and comparison with von Willebrand factor. Mod Pathol 1994;7:82-90.

19. Bhansali S, Dutta P, Kumar V, et al. Efficacy of autologous bone marrow-derived mesenchymal stem cell and mononuclear cell transplantation in type 2 diabetes mellitus: a randomized, placebo-controlled comparative study. Stem Cells Dev 2017;26:471-81.

20. Guan LX, Guan H, Li HB, et al. Therapeutic efficacy of umbilical cord-derived mesenchymal stem cells in patients with type 2 diabetes. Exp Ther Med 2015;9:1623-30.

21. Chow L, Johnson V, Coy J, et al. Mechanisms of immune suppression utilized by canine adipose and bone marrowderived mesenchymal stem cells. Stem Cells Dev 2017;26:374-89.

22. Lin CS, Lin G, Lue TF. Allogeneic and xenogeneic transplantation of adipose-derived stem cells in immunocompetent recipients without immunosuppressants. Stem Cells Dev 2012;21:2770-8.
23. Lasso JM, Perez Cano R, Castro Y, et al.

Xenotransplantation of human adipose-derived stem cells in the regeneration of a rabbit peripheral nerve. J Plast Reconstr Aesthet Surg 2015;68:e189-97.

24. Lee SY, Kwon B, Lee K, et al. Therapeutic mechanisms of human adipose-derived mesenchymal stem cells in a rat tendon injury model. The American journal of sports medicine 2017;45:1429-39.

25. Razavi S, Ghasemi N, Mardani M, et al. Cotransplantation of human neurotrophic factor secreting cells and adipose-derived stem cells in rat model of multiple sclerosis. Cell journal 2018;20:46-52.

26. Kuismanen K, Juntunen M, Narra Girish N, et al. Functional outcome of human adipose stem cell injections in rat anal sphincter acute injury model. Stem Cells Transl Med 2018;7:295-304.

27. Caetano G, Wang W, Murashima A, et al. Tissue constructs with human adipose-derived mesenchymal stem cells to treat bone defects in rats. Materials (Basel) 2019;12:2268.

28. Xie X, Wang Y, Zhao C, et al. Comparative evaluation of MSCs from bone marrow and adipose tissue seeded in PRP-derived scaffold for cartilage regeneration. Biomaterials 2012;33:7008-18.

29. Horie M, Choi H, Lee RH, et al. Intra-articular injection of human mesenchymal stem cells (MSCs) promote rat meniscal regeneration by being activated to express Indian hedgehog that enhances expression of type II collagen. Osteoarthritis Cartilage 2012;20:1197-207.

30. McKinney JM, Doan TN, Wang L, et al. Therapeutic efficacy of intra-articular delivery of encapsulated human mesenchymal stem cells on early stage osteoarthritis. Eur Cell Mater 2019;37:42-59.

31. Li M, Luo X, Lv X, et al. In vivo human adipose-derived mesenchymal stem cell tracking after intra-articular delivery in a rat osteoarthritis model. Stem Cell Res Ther 2016;7:160.

32. Grigolo B, Lisignoli G, Desando G, et al. Osteoarthritis treated with mesenchymal stem cells on hyaluronanbased scaffold in rabbit. Tissue Eng Part C Methods 2009; 15:647-58.

33. Sato M, Uchida K, Nakajima H, et al. Direct transplantation of mesenchymal stem cells into the knee joints of Hartley strain guinea pigs with spontaneous osteoarthritis. Arthritis Res Ther 2012;14:R31.

34. Mancuso P, Raman S, Glynn A, et al. Mesenchymal stem cell therapy for osteoarthritis: the critical role of the cell secretome. Front Bioeng Biotechnol 2019;7:9. 
35. Liu X, Duan B, Cheng Z, et al. SDF-1/CXCR4 axis modulates bone marrow mesenchymal stem cell apoptosis, migration and cytokine secretion. Protein Cell 2011;2:845-54.

doi: $10.21037 /$ sci-2020-003

Cite this article as: Wang Z, Zhu H, Dai S, Liu K, Ge C. Alleviation of medial meniscal transection-induced osteoarthritis pain in rats by human adipose derived mesenchymal stem cells. Stem Cell Investig 2020;7:10.
36. Namba A, Aida Y, Suzuki N, et al. Effects of IL-6 and soluble IL-6 receptor on the expression of cartilage matrix proteins in human chondrocytes. Connect Tissue Res 2007;48:263-70. 